\title{
Phonoritons as Hybridized Exciton-Photon-Phonon Excitations in a Monolayer $h$-BN Optical Cavity
}

\author{
Simone Latini $\odot,{ }^{1, *}$ Umberto De Giovannini ${ }^{1,2, \dagger}$ Edbert J. Sie ${ }^{3,4}$ \\ Nuh Gedik, ${ }^{3}$ Hannes Hübener(1), ${ }^{1,}$ and Angel Rubio ${ }^{1,2,5, \S}$ \\ ${ }^{1}$ Max Planck Institute for the Structure and Dynamics of Matter and Center for Free Electron Laser Science, \\ 22761 Hamburg, Germany \\ ${ }^{2}$ Nano-Bio Spectroscopy Group, Departamento de Fisica de Materiales, Universidad del Pas Vasco UPV/EHU, \\ 20018 San Sebastin, Spain \\ ${ }^{3}$ Department of Physics, Massachusetts Institute of Technology, Cambridge, Massachusetts 02139, USA \\ ${ }^{4}$ Geballe Laboratory for Advanced Materials, Stanford University, Stanford, California 94305, USA \\ ${ }^{5}$ Center for Computational Quantum Physics (CCQ), The Flatiron Institute, \\ 162 Fifth avenue, New York, New York 10010, USA
}

(Received 24 November 2020; accepted 2 April 2021; published 4 June 2021)

\begin{abstract}
A phonoriton is an elementary excitation that is predicted to emerge from hybridization between exciton, phonon, and photon. Besides the intriguing many-particle structure, phonoritons are of interest as they could serve as functional nodes in devices that utilize electronic, phononic, and photonic elements for energy conversion and thermal transport applications. Although phonoritons are predicted to emerge in an excitonic medium under intense electromagnetic wave irradiation, the stringent condition for their existence has eluded direct observation in solids. In particular, on-resonance, intense pumping schemes have been proposed, but excessive photoexcitation of carriers prevents optical detection. Here, we theoretically predict the appearance of phonoritonic features in monolayer hexagonal boron nitride $(h$-BN) embedded in an optical cavity. The coherent superposition nature of phonoriton states is evidenced by the hybridization of exciton-polariton branches with phonon replicas that is tunable by the cavity-matter coupling strength. This finding simultaneously provides an experimental pathway for observing the predicted phonoritons and opens a new avenue for tuning materials properties.
\end{abstract}

DOI: 10.1103/PhysRevLett.126.227401

New states of matter can arise from hybridization of fundamental modes of solid state systems with light. When the light-matter interaction is strong, for instance through confinement of the photons in optical resonators or cavities, these states can be described as bosonic quasiparticles, polaritons. There has been a resurgence of interest in the change of the properties in the material that host the polariton inside the cavity. Especially changes to the electron-phonon coupling, and hence, the possibility to affect superconducting properties of materials in optical cavities with strong coupling are being theoretically explored, predicting an enhancement of the transition temperature [1], control of electron-phonon parameter [2], or even an altogether new electron pairing mechanism through cavity photons [3], while first experimental evidence points toward the possibility to enhance the superconducting gap through cavity

Published by the American Physical Society under the terms of the Creative Commons Attribution 4.0 International license. Further distribution of this work must maintain attribution to the author(s) and the published article's title, journal citation, and DOI. Open access publication funded by the Max Planck Society. coupling [4]. Among other features that have been observed for materials in strongly coupled cavities are enhanced exciton lifetimes [5-7], theoretical predictions for controlling of exciton ordering [8], and an exciton-insulator superradiance phase [9], as well as the possibility to control the para- to ferroelectric phase transition in $\mathrm{SrTiO}_{3}[10,11]$. Finally, the possibility to break time-reversal symmetry with circular polarized cavity photons has been suggested to allow control of topological properties [12]. These recent developments suggest the emergence of the field of cavity materials engineering.

When changing materials properties with cavities, one can, in principle, couple any elementary excitation of the solid to the cavity photons, in accordance with the known "zoo" of solid state polaritons [13], including exciton polaritons and phonon polaritons. Even though such excitations occur in real materials at very different energy scales, through the coupling to a cavity, they can be tuned into mutual resonance. An example of a three-component polarization quasiparticle as the hybridization between exciton, phonon, and photon has been predicted by Ivanov and Keldysh [14] to emerge in a semiconductor under intense electromagnetic radiation tuned at the exciton 


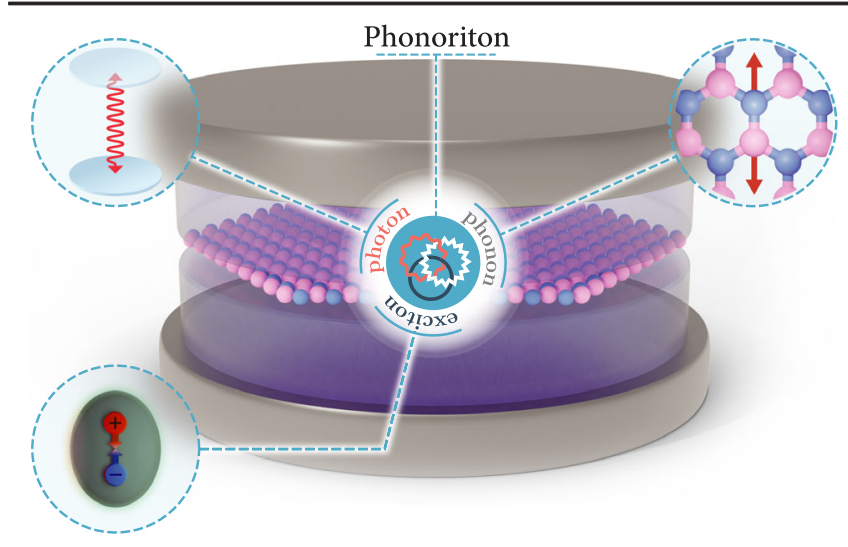

FIG. 1. The phonoriton is a hybridization between excitons, phonons, and photons that can be realized by embedding a crystal in an optical cavity.

resonance. The authors called this new quasiparticle a phonoriton to highlight its status as a fundamental mode on par with polaritons. However, despite the theoretical proposal, there has been little experimental evidence, except for a few reports [15-17], that implicitly invoked this phenomena, because direct observation in laser pumped systems is experimentally challenging. In particular, the on-resonance, intense pumping scheme leads to excessive photoexcitation of carriers that prevents the optical detection and may possibly damage the materials before the phonoritons start to emerge. By contrast, in a cavity, the strong light-matter interaction is not achieved via intensity of the light but, instead, by an enhanced coupling strength that results from confinement of the photon modes.

Here, we predict, from first principles, the appearance of such a three-component composite quasiparticle in a monolayer $h$-BN embedded in an optical cavity and discuss its realization in other 2D materials. By combining many-body perturbation theory and the quantum electrodynamical description of the exciton polariton [8], we show that phonoriton states occur when Rabi splitting of the polariton comes into resonance with phonon modes of the material. Such states are neither purely excitonic nor phononic, but the presence of the cavity photons leads to a mixing of all three modes as sketched in Fig. 1. We argue that the realization of this proposal gives, in fact, a novel view on the realities of materials in optical cavities and the prospective control and design of their properties. The tunability of elementary modes by dressing with light allows us to create new particle hybridizations and provides an opportunity to explore new directions of materials research utilizing strong light-matter interaction as well as providing a fruitful platform to rethink fundamental excitations in solids.

To describe the dressing of excitons by cavity photons from first principles, we consider the fundamental manybody Hamiltonian of mutually interacting electrons and photons in (nonrelativistic) quantum electrodynamics (QED). For a single photonic mode of energy $\omega_{c}$ in the velocity gauge representation and within the dipole approximation, reads

$$
\begin{aligned}
\hat{H}_{\mathrm{QED}}= & \hat{H}_{\mathrm{el}}+\omega_{c} \hat{b}^{\dagger} \hat{b}+\frac{A_{0}^{2}}{2}\left(\hat{b}^{\dagger}+\hat{b}\right)^{2} \\
& +A_{0} \sum_{r s \mathbf{k}}\left(\left\langle\phi_{r \mathbf{k}}|\hat{e} \cdot \hat{p}| \phi_{s \mathbf{k}}\right\rangle \hat{c}_{r \mathbf{k}}^{\dagger} \hat{c}_{s \mathbf{k}} \hat{b}^{\dagger}+\text { H.c. }\right),
\end{aligned}
$$

where $\hat{b}^{\dagger}$ and $\hat{b}$ are the photon creation and annihilation operators, $\hat{H}_{\mathrm{el}}$ the many-body electronic Hamiltonian, $\hat{c}_{r \mathbf{k}}^{\dagger}$, $\hat{c}_{s \mathbf{k}}$ the electronic creation and annihilation operators (with $r, s$ band indices and $\mathbf{k}$ wave vectors in the first Brillouin zone), $\hat{p}$ the single particle momentum operator, $\phi$ the single electron wave functions, $\hat{e}$ the photon field polarization, and $A_{0}$ the amplitude of the vector potential. The generalization to a multimode setting is straightforward and can be found in Ref. [8].

To describe the effect of lattice motion and introduce the interaction with phonons, we apply linear response theory and expand both the electronic and the light-matter term in the QED Hamiltonian in terms of the phononic displacement coordinates. By considering phonons $\alpha$ of energy $\Omega_{\alpha}$ and retaining the terms up to first order in the phonon displacement, the standard exciton-phonon coupling emerges as well as a three-way quasiparticle coupling from the exciton-photon term, a full derivation is provided in the Supplemental Material (SM) [18]. Since the coupling of photons to the electronic degrees of freedom involves the creation or annihilation of neutral electron-hole pairs, it is natural to approximate the many-body eigenstates of the electronic Hamiltonian by exciton states, as done in Ref. [8], i.e., $\hat{H}_{\mathrm{el}}\left|\Psi_{i}^{\text {exc }}\right\rangle \simeq \epsilon_{i}^{\text {exc }}\left|\Psi_{i}^{\text {exc }}\right\rangle$. The phonon perturbed Hamiltonian, including the free phonon energy, reads in the excitonic basis

$$
\begin{aligned}
& \left\langle\Psi_{i}^{\operatorname{exc}}|\hat{H}| \Psi_{j}^{\operatorname{exc}}\right\rangle \\
& =\left[\epsilon_{i}^{\operatorname{exc}}+\omega_{c} \hat{b}^{\dagger} \hat{b}+\sum_{\alpha} \Omega_{\alpha} \hat{a}_{\alpha}^{\dagger} \hat{a}_{\alpha}+\frac{A_{0}^{2}}{2}\left(\hat{b}^{\dagger}+\hat{b}\right)^{2}\right] \delta_{i j} \\
& \quad+\sum_{\alpha}\left(\mathcal{G}_{i j, \alpha}^{\operatorname{exc}} \hat{a}_{\alpha}^{\dagger}+\mathcal{G}_{j i, \alpha}^{\operatorname{exc} *} \hat{a}_{\alpha}\right)+A_{0}\left(\mathcal{M}_{i j}^{\operatorname{exc}} \hat{b}^{\dagger}+\mathcal{M}_{j i}^{\operatorname{exc} *} \hat{b}\right) \\
& \quad+A_{0} \sum_{\alpha}\left(\mathcal{Z}_{i j, \alpha}^{\operatorname{exc}} \hat{b}^{\dagger}+\mathcal{Z}_{j i, \alpha}^{\operatorname{exc} *} \hat{b}\right)\left(\hat{a}_{\alpha}^{\dagger}+\hat{a}_{\alpha}\right)
\end{aligned}
$$

where $\hat{a}_{\alpha}^{\dagger}$ and $\hat{a}_{\alpha}$ are the phonon (photon) creation and annihilation operators, respectively, $\mathcal{M}_{i j}^{\text {exc }}=$ $\left\langle\Psi_{i}^{\text {exc }}\left|\sum_{r s \mathbf{k}}\left\langle\phi_{r \mathbf{k}}|\hat{e} \cdot \hat{p}| \phi_{s \mathbf{k}}\right\rangle \hat{c}_{r \mathbf{k}}^{\dagger} \hat{c}_{s \mathbf{k}}\right| \Psi_{j}^{\text {exc }}\right\rangle$ are the excitonphoton matrix elements, $\mathcal{G}_{i j, \alpha}^{\text {exc }}=\left\langle\Psi_{i}^{\text {exc }}\right| \sum_{r s \mathbf{k}} g_{r s \mathbf{k}}^{\alpha} \hat{c}_{r \mathbf{k}}^{\dagger} \times$ $\hat{c}_{s \mathbf{k}}\left|\Psi_{j}^{\mathrm{exc}}\right\rangle$ are the exciton-phonon matrix elements with $g$ the standard electron-phonon matrix elements, and $\mathcal{Z}_{i j}^{\text {exc }}=$ $\sqrt{\left(1 / 2 M_{\alpha} \Omega_{\alpha}\right)}\left\langle\Psi_{i}^{\text {exc }}\left|\sum_{r s \mathbf{k}}\left(\partial / \partial \mathbf{R}_{\alpha}\right)\left\langle\phi_{r \mathbf{k}}|\hat{e} \cdot \hat{p}| \phi_{s \mathbf{k}}\right\rangle \hat{c}_{r \mathbf{k}}^{\dagger} \hat{c}_{s \mathbf{k}}\right|\right.$ $\left.\Psi_{j}^{\text {exc }}\right\rangle$, with $\mathbf{R}_{\alpha}$ phonon displacement, describe the coupling between phonons, photons, and excitons, and therefore, we 
refer to $\mathcal{Z}$ as the phonoriton matrix element. The Hamiltonian above is the central quantity of this Letter, and its eigenstates are the phonoriton quasiparticle states, $\Psi_{I}^{\mathrm{p}}$ discussed below, with $I$ the phonoriton index. The formation of a phonoriton can be understood, in simple terms, as the photon dressing of an exciton-phonon system which results in a new interaction term that couples the exciton and the phonons beyond the standard excitonphonon coupling (see Fig. S1 in the SM [18]). We stress that, differently from the model of the original proposal [14,31], here, we do not involve scattering of higher momentum excitons and phonons. We explicitly account for the photon-phonon interaction only through the exciton since the relevant modes that we consider for the following phonoritonic features are not optically active.

The Hamiltonian in Eq. (2) can be numerically diagonalized in a mixed exciton-photon-phonon product-state basis $\left|\Psi_{i}^{\text {exc }}\right\rangle \otimes|n\rangle \otimes|m\rangle$, where $|n\rangle$ and $|m\rangle$ are phonon and photon number states, respectively. We set up the Hamiltonian for the specific case of monolayer $h$-BN, whose phonons have been extensively investigated in the case of phonon-polariton formation [32-34]. To give a clear account of the mechanism driving the creation of the phonoriton, here, we simplify the calculations by including the lowest excitonic state and by considering only the zeromomentum optical phonon modes. The in-plane modes in $h$-BN are usually denoted as longitudinal (LO) and transverse (TO) modes indicating their contribution to a macroscopic polarization field, however, at the Brillouion zone center, they are degenerate at $\sim 166 \mathrm{meV}$. The out-of-plane mode (ZO) has an energy of $\sim 96 \mathrm{meV}$. Given the high energy of such phonons, we neglect, in the following, the effect of thermal population. The excitons and phonons are calculated from first principles: for the exciton, we solved the Bethe-Salpeter equation using the GPAW code $[19,20]$, while the electron-phonon matrix elements were computed with the Octopus code [21]. From this, we are able to calculate the matrix elements $\mathcal{M}_{m n}^{\mathrm{exc}}, \mathcal{G}_{i j, \alpha}^{\mathrm{exc}}$, and $\mathcal{Z}_{m n}^{\mathrm{exc}}$ in the Hamiltonian of Eq. (2) on a first principles level, for the specific case of monolayer $h$-BN. For the phonoritonic matrix elements, we assume that the envelope function of the exciton is, to first order, unchanged, and we only account for the variation of the conduction-valence matrix elements as a function of lattice displacement. For the photons, we consider the first nonzero photon mode, $\omega_{c}=\pi c / L_{\perp}$, which has an out-of-plane wave vector and in-plane electric field and, therefore, is able to couple to the $h$-BN in-plane excitonic dipole. Changing $L_{\perp}$, the vertical dimension of the cavity, allows for the tuning of the cavity frequency. This can be done by, for instance, adding an optically inactive spacer material. The quasi-2D cavity configuration is characterized by a frequency independent vector potential amplitude which, for a perfect cavity with an embedding dielectric medium with dielectric constant $\epsilon=1$, is $A_{0}$, i.e., $A_{0}=1 / \sqrt{a L_{\perp} \omega_{c}}=1 / \sqrt{2 \pi c a}$, where $a$ is the unit cell area of $h$-BN. In the following, we investigate the effect of cavity-matter coupling by using $A_{0}$ as a tuning parameter.

Results.-In order to unravel the spectral features of the phonoriton, we calculate the matter component of the full optical response $\chi\left(\omega_{c}, \omega_{\text {probe }}\right)$, a quantity which can be experimentally measured. Within linear response theory, the matter response is given by [35,36] $\chi\left(\omega_{c}, \omega_{\text {probe }}\right)=$ $\sum_{I}\left[\left(\mathcal{M}_{0 I}^{\mathrm{p}} \mathcal{M}_{I 0}^{\mathrm{p}}\right) /\left(\omega_{\text {probe }}-E_{I}^{\mathrm{p}}\left(\omega_{c}\right)+E_{0}^{\mathrm{p}}\left(\omega_{c}\right)+i \eta\right)\right]$, where $\mathcal{M}_{I J}^{\mathrm{p}}=\left\langle\Psi_{I}^{\mathrm{p}}|\hat{p}| \Psi_{J}^{\mathrm{p}}\right\rangle$, and $\eta$ is an artificial broadening which, in the following, is kept fixed but can, in principle, be tuned to reproduce thermal broadening of the spectral features.

Figure 2 reports the $a b$ initio optical response of $h$-BN embedded in a cavity with tunable frequency $\omega_{c}$ and coupling strength $A_{0}=0.02$ a.u.. Here, one can observe the appearance of a strong optical signal between the lower and upper exciton-polariton branches, as compared to the bare exciton-polariton case [cf. inset Fig. 2(a)]. The additional spectral features show the presence of the phononic series that interferes with the upper exciton-polariton branch yielding a strong signal modulation that we identify as phonoritonic resonances.

The formation of phonoritons opens up new optical transition channels for the phonon sidebands that are, otherwise, dipole forbidden in the uncoupled states because all excitations couple to the same cavity photon. The cavity

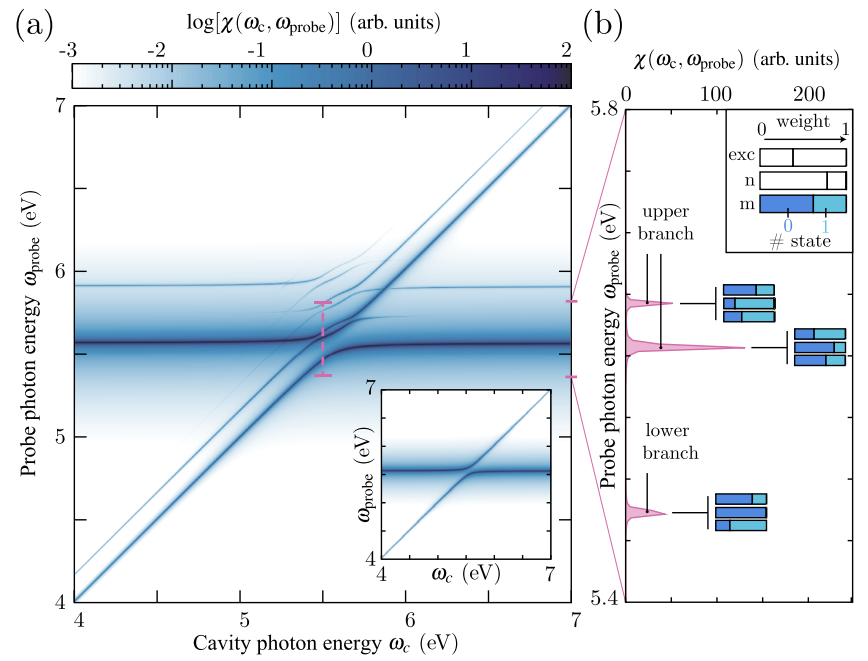

FIG. 2. Computed optical spectrum of a monolayer $h-\mathrm{BN}$ in a strongly coupled optical cavity as a function of cavity energy $\omega_{c}$ for the coupling strength $A_{0}=0.02$ a.u.. The optical response is shown in logarithmic scale, $\log [\chi]$. The characteristic avoided crossing of an exciton polariton is modulated by the additional hybridization of phonon modes in the material, giving composite states of exciton-photon-phonon character. The inset shows the exciton-polariton response in absence of phononic and phonoritonic coupling. The dashed line indicates a cut through the spectrum that is analyzed in panel (b): each of these peaks are transitions from the ground state to an excited state of the phonoriton Hamiltonian (see text). 
photon acts as a glue introducing further coupling between excitons and phonon modes. We note that the direct excitonphonon coupling adds spectral features associated with phonon replicas, at energies above the upper exciton-polariton branch, which are not as intense as the phonoriton signal and, indeed, can only be observed in logarithmic scale.

Analyzing the $a b$ initio matrix elements in the Hamiltonian, Eq. (2), we can single out the LO mode as the one responsible for the formation of phonoritons in $h$-BN, because it is the only phonon which has a nonzero phonoriton matrix element, $\mathcal{Z}_{\mathrm{LO}}=-0.02+$ $0.014 i$ a.u.. For the exciton-phonon coupling, both LO and $\mathrm{TO}$ modes have similar values $\left(\mathcal{G}_{\mathrm{LO}}=0.004+\right.$ $0.002 i$ a.u., $\mathcal{G}_{\mathrm{TO}}=0.002+0.004 i$ a.u.) while the $\mathrm{ZO}$ mode has a negligible value. Since the TO mode does not contribute to the phonoriton and is degenerate with the LO mode, it only contributes to the intensity of the exciton-phonon spectral signal and, therefore, is omitted in the calculations. Instead, the exciton-photon coupling is $\mathcal{M}_{01}=0.06+0.10 i$ a.u.

To disentangle which mixing channels are enabled by the cavity, we have dissected the contribution of the different particles, exciton, phonons, and photons, to the phonoritonic features that are discernible in a linear scale, for a cavity frequency of $\omega_{c}=5.5 \mathrm{eV}$, cf. Fig. 2(b). To quantify the degree of hybridization, we define a particle weight by taking the square of the phonoritonic state $\Psi_{I}^{\mathrm{p}}$ responsible of a given spectral feature and trace out all the degrees of freedom, except for one kind of particle. For example, the excitonic weight is computed as $w_{I}^{\mathrm{exc}}=\sum_{n m}\left|\Psi_{I, i n m}^{\mathrm{p}}\right|^{2}$, and, similarly, for the LO phonon mode and for the cavity photon, shown in Fig. 2(b). States in different polaritonic branches are characterized by different excitonic, phononic, and photonic content: the lower branch is mainly made of the ground electronic state and one photon, while the upper branch, which is split in two, contains excitonic states as well as both phononic and photonic number states up to $n, m=1$. This peak splitting reveals the formation of a phonoriton. In particular, the peak at $\omega_{\text {probe }} \approx 5.61 \mathrm{eV}$ has a major contribution of the excitonic state and the $n, m=0$ phononic and photonic states, while the peak at $\omega_{\text {probe }} \approx 5.64 \mathrm{eV}$ is mainly made by the ground electronic state and the $n, m=1$ phononic and photonic states.

The fact that the upper polaritonic branch participates in the phonoriton formation hints at the great potential of tunability of the phonoritonic response. Indeed, by changing the cavity frequency, the Rabi splitting can be tuned to a different phonon number or, in more complex materials, to other phonon modes to selectively involve them in the phonoriton formation. We stress that the coupling strength is within experimental reach as demonstrated in Ref. [37], and we have shown in Ref. [8] that the cavity parameters can be tuned, for example, by changing the size of the device and/or altering the dielectric environment. Finally, to

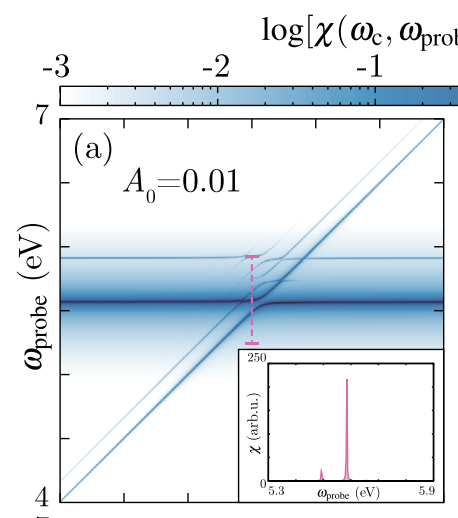

] (arb. units)

1

\section{1} 2
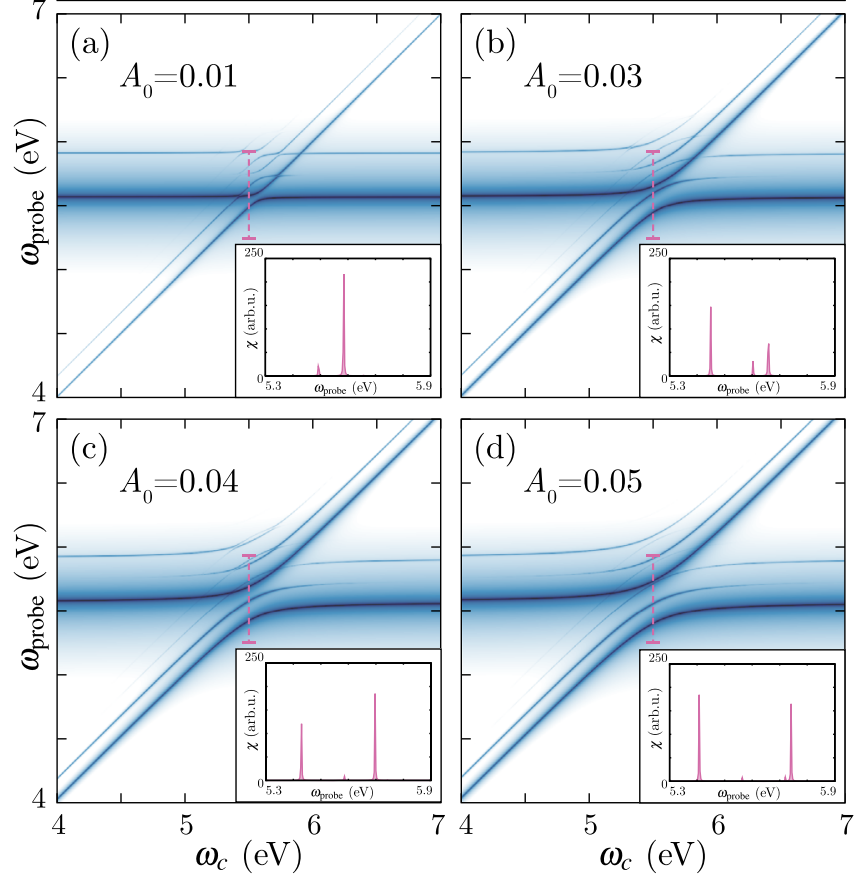

FIG. 3. Computed optical spectrum of a monolayer $h-\mathrm{BN}$ in a strongly coupled optical cavity as a function of cavity energy $\omega_{c}$ for different coupling strength $A_{0}$ in atomic units and in logarithmic scale. Dashed lines indicate cuts that correspond to the spectra shown in linear scale in the insets.

highlight the role of cavity-matter coupling and, hence, the Rabi splitting in the realization of the phonoritonic states, we calculated the optical response of $h$-BN for different coupling strengths, cf. Fig. 3. Somewhat counterintuitively, a stronger coupling does not necessarily enhance the phonoriton, but instead, the comparison of the different couplings in Fig. 3 and Fig. 2(a) show that, for a clear phonoriton signal, the phonon line needs to be close to resonance with the Rabi splitting, and since low phonon number states have a stronger phonoritonic coupling, a large Rabi splitting can wash out the phonoriton features.

The phonoriton features described here rely on a phonoritonic coupling term that is not negligible when compared to the exciton-photon coupling. Because of mass dependence of the phonoritonic coupling (see definition), it is expected, as shown for $h$-BN, that crystals composed of lighter atoms are more likely to host phonoritonic quasiparticles. This is confirmed by calculations for the transition metal dichalcogenide $\mathrm{WS}_{2}$, which has heavier elements than $h$ - BN, where we have found the phonoriton features to be much less pronounced.

A relevant experimental challenge for the realization of our proposal is the fabrication of a cavity whose photon energy is high enough to be in resonance with the exciton in $h$-BN. Indeed, a Fabry-Perot type of cavity, with a 
fundamental mode of $5.5 \mathrm{eV}$, would require a distance between the mirrors of $225 \mathrm{~nm}$. However, it is feasible to employ higher cavity modes of existing cavity designs in the IR range [38]. This approach would be the equivalent to using higher harmonics of a laser. The theory reported above directly extends to the case of higher modes as long as the $A_{0}$ is properly scaled as $A_{0} \propto 1 / \sqrt{n}$ where $n$ is the mode number, under the assumption that the cavity modes are independent, as shown in Ref. [8]. Another aspect to consider is the choice of the encapsulating dielectric material, such that it does not interact with the cavity (UV) photons, possible candidates are oxides like $\mathrm{HfO}_{2}$. An alternative pathway toward the realization of phonoritonic states could be to change the host material to one with a smaller band gap that has a small phonon effective mass and retains strong excitonic features in the visible range: graphane [39] and graphene oxide are promising candidates. Using two different layers as active material is yet another option, where one provides the excitons and the other one provides the phonons: a possible candidate with such features is the $\mathrm{WSe}_{2}-h$-BN bilayer which shows a strong coupling of the $\mathrm{WSe}_{2}$ exciton with the $\mathrm{ZO}$ phonons of $h$-BN [40]. Finally, while the presented phonoritonic description is explicated for the case of zero momentum particles, it can be directly extended to finite momentum and be, for example, used to investigate the potential of highly tunable surface acoustic waves for the formation of phonoritons [41-43].

Conclusion.-We have proposed a general first-principle framework to account for lattice motion effects in the QED Hamiltonian, and we rediscovered a three-way quasiparticle, the phonoriton. We have predicted the effect of this exciton-phonon-photon quasiparticle on the optical response of monolayer $h-\mathrm{BN}$ in an optical cavity. The standard exciton-polariton branch picture is enriched by bright features originating from hybridized phonon states which are glued together by the photon of the cavity. We have also shown that, via the cavity-matter coupling strength, one can potentially control the ingredients of the phonoriton by involving different phonons or other modes, condensate phonoritons, or realize chiral phonoritons in chiral cavities [12].

We thank Emre Ergecen for constructive discussions. We acknowledge financial support from the European Research Council (Grant No. ERC-2015-AdG-694097). Grupos Consolidados (Grant No. IT1249-19), and the Cluster of Excellence "CUI: Advanced Imaging of Matter" of the Deutsche Forschungsgemeinschaft (DFG)—EXC 2056Project ID No. 390715994. The Flatiron Institute is a division of the Simons Foundation. S. L. acknowledges support from the Alexander von Humboldt foundation. Work at MIT was supported by the US Department of Energy, BES DMSE and by the Gordon and Betty Moore Foundations EPiQS Initiative Grant No. GBMF9459. *simone.latini@mpsd.mpg.de umberto.degiovannini@gmail.com

*hannes.huebener@gmail.com

§angel.rubio@mpsd.mpg.de

[1] J. B. Curtis, Z. M. Raines, A. A. Allocca, M. Hafezi, and V. M. Galitski, Phys. Rev. Lett. 122, 167002 (2019).

[2] M. A. Sentef, M. Ruggenthaler, and A. Rubio, Sci. Adv. 4, eaau6969 (2018).

[3] F. Schlawin, A. Cavalleri, and D. Jaksch, Phys. Rev. Lett. 122, 133602 (2019).

[4] A. Thomas, E. Devaux, K. Nagarajan, T. Chervy, M. Seidel, D. Hagenmller, S. Schtz, J. Schachenmayer, C. Genet, G. Pupillo, and T. W. Ebbesen, arXiv:1911.01459.

[5] Y.-J. Chen, J. D. Cain, T. K. Stanev, V. P. Dravid, and N. P. Stern, Nat. Photonics 11, 431 (2017).

[6] Z. Sun, J. Gu, A. Ghazaryan, Z. Shotan, C. R. Considine, M. Dollar, B. Chakraborty, X. Liu, P. Ghaemi, S. Kéna-Cohen, and V. M. Menon, Nat. Photonics 11, 491 (2017).

[7] S. Dufferwiel, T. P. Lyons, D. D. Solnyshkov, A. A. P. Trichet, F. Withers, S. Schwarz, G. Malpuech, J. M. Smith, K. S. Novoselov, M. S. Skolnick, D. N. Krizhanovskii, and A. I. Tartakovskii, Nat. Photonics 11, 497 (2017).

[8] S. Latini, E. Ronca, U. De Giovannini, H. Hübener, and A. Rubio, Nano Lett. 19, 3473 (2019).

[9] G. Mazza and A. Georges, Phys. Rev. Lett. 122, 017401 (2019).

[10] Y. Ashida, A. Imamoglu, J. Faist, D. Jaksch, A. Cavalleri, and E. Demler, Phys. Rev. X 10, 041027 (2020).

[11] S. Latini, D. Shin, S. A. Sato, C. Schäfer, U. De Giovannini, H. Hübener, and A. Rubio, arXiv:2101.11313.

[12] H. Hübener, U. de Giovannini, C. Schäfer, J. Andberger, M. Ruggenthaler, J. Faist, and A. Rubio, Nat. Mater. 20, 438 (2021).

[13] D. N. Basov, M. M. Fogler, and F. J. Garcia de Abajo, Science 354, aag1992 (2016).

[14] L. V. Keldysh, JETP Lett. 29, 658 (1979), https://ui.adsabs .harvard.edu/abs/1979JETPL..29..658K/abstract.

[15] G. Vygovskii, G. Golubev, E. Zhukov, A. Fomichev, and M. Yakshin, JETP Lett. 42, 164 (1985), https://ui.adsabs .harvard.edu/abs/1985ZhPmR..42..134V/abstract.

[16] B. I. Greene, J. F. Mueller, J. Orenstein, D. H. Rapkine, S. Schmitt-Rink, and M. Thakur, Phys. Rev. Lett. 61, 325 (1988).

[17] L. Hanke, D. Frohlich, A. L. Ivanov, P. B. Littlewood, and H. Stolz, Phys. Rev. Lett. 83, 4365 (1999).

[18] See Supplemental Material at http://link.aps.org/ supplemental/10.1103/PhysRevLett.126.227401 for the derivation of the main equations and the computational details, which include Refs. [8,19-30].

[19] J. J. Mortensen, L. B. Hansen, and K. W. Jacobsen, Phys. Rev. B 71, 035109 (2005).

[20] J. Enkovaara et al., J. Phys. Condens. Matter 22, 253202 (2010).

[21] N. Tancogne-Dejean et al., J. Chem. Phys. 152, 124119 (2020).

[22] F. Giustino, Rev. Mod. Phys. 89, 015003 (2017).

[23] S. Latini, T. Olsen, and K. S. Thygesen, Phys. Rev. B 92, 245123 (2015).

[24] M. Rohlfing and S. G. Louie, Phys. Rev. Lett. 81, 2312 (1998). 
[25] M. Rohlfing and S. G. Louie, Phys. Rev. B 62, 4927 (2000).

[26] G. Onida, L. Reining, and A. Rubio, Rev. Mod. Phys. 74, 601 (2002).

[27] L. Wirtz, A. Marini, M. Grüning, C. Attaccalite, G. Kresse, and A. Rubio, Phys. Rev. Lett. 100, 189701 (2008).

[28] X. Blase, A. Rubio, S. G. Louie, and M. L. Cohen, Phys. Rev. B 51, 6868 (1995).

[29] S. Galambosi, L. Wirtz, J. A. Soininen, J. Serrano, A. Marini, K. Watanabe, T. Taniguchi, S. Huotari, A. Rubio, and K. Hämäläinen, Phys. Rev. B 83, 081413(R) (2011).

[30] S. Haastrup, M. Strange, M. Pandey, T. Deilmann, P. S. Schmidt, N. F. Hinsche, M. N. Gjerding, D. Torelli, P. M. Larsen, A. C. Riis-Jensen, J. Gath, K. W. Jacobsen, J. J. Mortensen, T. Olsen, and K. S. Thygesen, 2D Mater. 5, 042002 (2018).

[31] B. S. Wang and J. L. Birman, Phys. Rev. B 42, 9609 (1990).

[32] S. Dai, Z. Fei, Q. Ma, A. Rodin, M. Wagner, A. McLeod, M. Liu, W. Gannett, W. Regan, K. Watanabe et al., Science 343, 1125 (2014).

[33] T. Low, A. Chaves, J. D. Caldwell, A. Kumar, N. X. Fang, P. Avouris, T. F. Heinz, F. Guinea, L. Martin-Moreno, and F. Koppens, Nat. Mater. 16, 182 (2017).

[34] J. Serrano, A. Bosak, R. Arenal, M. Krisch, K. Watanabe, T. Taniguchi, H. Kanda, A. Rubio, and L. Wirtz, Phys. Rev. Lett. 98, 095503 (2007).
[35] J. Flick, D. M. Welakuh, M. Ruggenthaler, H. Appel, and A. Rubio, ACS Photonics 6, 2757 (2019).

[36] M. Ruggenthaler, N. Tancogne-Dejean, J. Flick, H. Appel, and A. Rubio, Nat. Rev. Chem. 2, 0118 (2018).

[37] L. C. Flatten, Z. He, D. M. Coles, A. A. P. Trichet, A. W. Powell, R. A. Taylor, J. H. Warner, and J. M. Smith, Sci. Rep. 6, 33134 (2016).

[38] X. Liu, T. Galfsky, Z. Sun, F. Xia, E.-c. Lin, Y.-H. Lee, S. Kéna-Cohen, and V. M. Menon, Nat. Photonics 9, 30 (2015).

[39] D. C. Elias, R. R. Nair, T. M. G. Mohiuddin, S. V. Morozov, P. Blake, M. P. Halsall, A. C. Ferrari, D. W. Boukhvalov, M. I. Katsnelson, A. K. Geim, and K. S. Novoselov, Science 323, 610 (2009).

[40] C. Jin, J. Kim, J. Suh, Z. Shi, B. Chen, X. Fan, M. Kam, K. Watanabe, T. Taniguchi, S. Tongay, A. Zettl, J. Wu, and F. Wang, Nat. Phys. 13, 127 (2017).

[41] K. Cho, K. Okumoto, N. I. Nikolaev, and A. L. Ivanov, Phys. Rev. Lett. 94, 226406 (2005).

[42] E. A. Cerda-Méndez, D. N. Krizhanovskii, M. Wouters, R. Bradley, K. Biermann, K. Guda, R. Hey, P. V. Santos, D. Sarkar, and M. S. Skolnick, Phys. Rev. Lett. 105, 116402 (2010).

[43] E. A. Cerda-Méndez, D. Sarkar, D. N. Krizhanovskii, S. S. Gavrilov, K. Biermann, M. S. Skolnick, and P. V. Santos, Phys. Rev. Lett. 111, 146401 (2013). 